\title{
Desarrollo de la competencia comunicativa a través de la comunicación radiofónica en la clase de $\mathrm{E} / \mathrm{LE}$
}

\author{
AMPARO RODRIGO-MATEU \\ Universitat de València \\ amroma@alumni.uv.es
}

Resumen: Este artículo describe una experiencia práctica con estudiantes de E/LE de la Universidad de Mumbai, en India ${ }^{1}$. El objetivo es profundizar en el desarrollo de la competencia comunicativa a partir de la creación de contenidos radiofónicos. En él se describen las fases de desarrollo del proyecto, teniendo en cuenta tanto el contexto y la situación de aprendizaje, como las expectativas previas y las evaluaciones posteriores de los alumnos.

Palabras clave: Competencia comunicativa, competencia pragmática, español L2, expresión oral, radio.

\section{Development of Communicative Competence through Radio Communication in the Spanish as a Foreign Language Class}

Abstract: This article describes a classroom experience with learners of Spanish as a foreign language at the University of Mumbai, in India. The aim is to delve into the development of communicative competence through creation of radio contents. The different phases of the project are described, while considering learning and situation contexts, as well as previous expectations and subsequent evaluations from students.

Key words: Communicative competence, pragmatic competence, Spanish as foreign language, oral expression, radio.

\section{Introducción y objetivos}

Es frecuente encontrar, entre lecturas, una idea generalizada sobre la utilidad de recurrir a diálogos e interacciones, y a su reproducción o adaptación posteriores, como base para el desarrollo de la competencia pragmática en el aprendizaje de lenguas extranjeras. Entendemos aquí la competencia pragmática como componente inseparable de una competencia comunicativa más general y en coexistencia con la competencia léxicosemántica.

El objetivo de este artículo es avanzar un paso más y apoyar la idea de crear oportunidades en el aula para el desarrollo de la competencia pragmática, en lugar de enseñarla de manera explícita, es decir, facilitar que el aprendiz la descubra (Rodríguez 2004: 5), mediante la adaptación del uso de la lengua al contexto de comunicación. Intentamos, con ello, demostrar la viabilidad, la adecuación y la relevancia de este enfoque en la clase de español como lengua extranjera (en adelante, E/LE) de cara al

\footnotetext{
${ }^{1}$ Trabajo realizado en el contexto del programa Lectorados MAEC-AECID en Universidades Extranjeras. 
desarrollo de la competencia comunicativa en culturas lejanas, como es el caso de nuestro contexto de trabajo en la Universidad de Mumbai, en India.

Hablar del desarrollo de la competencia comunicativa nos refiere, necesariamente, también a la competencia lingüística $\mathrm{y}$, dentro de esta, a la observación de los procesos de adquisición de vocabulario, es decir, del desarrollo de la competencia léxica. Relacionado con esto, el Plan Curricular del Instituto Cervantes (2007) clasifica, con carácter orientativo, los contenidos léxicos en veinte temas o nociones específicas para los niveles A1 y A2, y añade lo siguiente:

En función del análisis que se haga de las necesidades y expectativas de los alumnos, así como de las características de cada situación particular de enseñanza, podrán añadirse nuevas entradas [...]. Los criterios de selección léxica vendrán, en todo caso, condicionados por las distintas variedades de la lengua [...] y en relación siempre con el entorno geográfico, social y cultural en el que se desarrolle el proceso de enseñanza y aprendizaje.

Instituto Cervantes (2007: 332-333).

Las indicaciones no incluyen organización didáctica, análisis gramaticales ni listas o estrategias para el aprendizaje de vocabulario. Son, más bien, una descripción de los objetivos de aprendizaje, basados en posibles contextualizaciones y diálogos reales. Con ello se entiende que los contenidos específicos de enseñanza, así como los criterios de selección, deben elegirse en función de las necesidades de los alumnos. Deducimos, por tanto, que la clasificación del léxico, más que criterios lingüísticos, sigue criterios comunicativos y de aplicación en situaciones reales de contexto nativo de la lengua.

Landone (2009), por su parte, habla del valor de la observación de situaciones comunicativas auténticas que permitan relacionar las formas lingüísticas y el pragmatismo. En este sentido, el lenguaje radiofónico puede ser un recurso útil en el aula de idiomas. Según Cassany (2016), ya en la fase de redacción del guion, los aprendientes deben adaptar el pragmatismo al contexto, atendiendo a aspectos como el propósito comunicativo, las convenciones sociales y discursivas del género, la modalidad de lengua utilizada y el registro apropiado. La escritura interviene como instrumento de reflexión, no solo para el aprendizaje de la gramática y del léxico, sino también de la pragmática.

Esta perspectiva pragmática que hemos querido destacar, -por considerarse inherente a la competencia comunicativa y que, en cierto modo, la determina- encuentra un reflejo en los trabajos de Escandell $(2004,2016)$, en los que se destacan aspectos como la interacción y la importancia de crear situaciones comunicativas en el aula. Esto aporta un enfoque práctico y proactivo de cara al desarrollo de la competencia pragmática, cuya evolución se ha pretendido explorar con el programa de radio que presentamos.

En cuanto al tipo de discurso, en el contexto radiofónico, el habla y la escritura interactúan en un discurso mixto $\mathrm{y}$, en la práctica de la locución, ambos se apoyan mutuamente (Cassany 2016). La información tiene que pasar, sin ninguna dificultad, de la oralidad a la escritura, primero, en el diseño del guion y, luego, de nuevo, a la oralidad en la locución. Esta confluencia de destrezas orales y escritas nos remite, obligatoriamente, a considerar la distinción entre lo que serían discursos orales espontáneos - característicos de la mayoría de las interacciones orales- y aquellos que suponen una planificación previa, como es el caso de los guiones radiofónicos. 


\begin{tabular}{|l|l|}
\hline \multicolumn{1}{|c|}{ Discurso oral planificado } & \multicolumn{1}{c|}{ Discurso oral espontáneo } \\
\hline Discurso preparado y ensayado. & Inmediatez en el tiempo y el espacio. \\
\hline $\begin{array}{l}\text { Relaciones personales determinadas de antemano } \\
\text { por un contexto dado y negociadas en la fase de } \\
\text { redacción del diálogo, previa a su representación. }\end{array}$ & $\begin{array}{l}\text { Negociación de las relaciones interpersonales a lo } \\
\text { largo del discurso. }\end{array}$ \\
\hline Mayor control de la producción y de la recepción. & Menor control de la producción y de la recepción. \\
\hline Autorreparaciones mínimas. & Autorreparaciones continuas. \\
\hline Se anticipa que el oyente va a comprender. & Se busca la comprensión del oyente. \\
\hline Mayor uso de la subordinación sintáctica. & Menor uso de la subordinación sintáctica. \\
\hline Mayor uso de marcadores discursivos. & Menor uso de marcadores discursivos. \\
\hline Léxico más elaborado y estudiado de antemano. & Léxico espontáneo. \\
\hline
\end{tabular}

Tabla 1. Diferencias principales entre los discursos orales planificados y espontáneos, Briz (2016: 221-222)

Para terminar, durante la práctica de locución, el grado de comprensión del guion previo determinará la calidad de la articulación del discurso radiofónico y, como apunta Rodero (en Blanch y Lázaro 2010: 163) también la velocidad. Esta última se podrá aumentar únicamente si se domina la articulación. Todo ello influirá en las pausas y en el significado que aportan al discurso. Blanch y Lázaro (2010) recomiendan que estas no excedan los tres segundos de duración.

\section{Metodología}

El programa radiofónico ¿Salsa o salsa? se ha realizado en el estudio de radio comunitaria Radio MUST, ubicado en la Universidad de Mumbai, durante el curso académico 2013-2014. El estudio consta de una sala para emisiones en directo, una sala de grabación y otra de control. Muchos programas se emiten en diferido y las ondas alcanzan un radio de $10 \mathrm{~km}$.

En la sección del programa que tratamos en este artículo ha participado un grupo de segundo curso de E/LE, de nivel A2 según Marco Común Europeo de Referencia para las lenguas (Ministerio de Educación, Cultura y Deporte 2002) (en adelante, MCER). Se trata de un total de 18 alumnos, con una asistencia irregular a clase aproximadamente en la mitad de ellos. Todos son estudiantes de español a tiempo parcial y tienen edades comprendidas entre los 22 y los 67 años. A partir de observaciones del trabajo en clase, se constata que cuentan con una base poco uniforme del idioma y esto les supone dificultades a la hora de utilizar expresiones y frases sencillas para describir lugares y personas familiares, lo que correspondería al nivel A1 inicial en expresión oral, según el MCER. Se trata, al mismo tiempo, de un grupo bastante cohesionado.

En la ideación del programa, la primera audiencia que se tiene en mente son oyentes indios que puedan escucharlo a través de las ondas de emisión de Radio MUST, es decir, una audiencia sin conocimientos de español. Esto requerirá una presentación y aclaración adecuadas y comprensibles de los contenidos lingüísticos y culturales. 
Asimismo, los programas se publican también en el blog de la asignatura ${ }^{2} \mathrm{y}$, por tanto, cualquier persona con acceso a Internet puede escucharlos.

La planificación del programa de radio empieza con la idea sobre cómo enfocar una propuesta de trabajo que despierte interés y curiosidad entre los alumnos y que, al mismo tiempo, se perciba como oportunidad de aprendizaje. Tras el planteamiento, los alumnos se sienten motivados, si bien algunos comentan abiertamente el miedo que les supone equivocarse. Es el miedo al error lo primero que les viene a la mente.

Empezamos con una lluvia de ideas sobre el tipo de programas que se podrían realizar. La creación de diálogos, la comparación entre el español y las lenguas locales y regionales en India y las fiestas que se celebran en el país asiático son algunas ideas que se proponen en esta primera aproximación. Resulta curioso y alentador comprobar que aspectos como la competencia comunicativa y el contraste lingüístico y cultural, ambos objetivos claros de antemano pero no compartidos directamente con los alumnos, surgen de sus mismas propuestas, por su propio interés y necesidades de aprendizaje. Podemos afirmar, por tanto, que la confluencia de intereses de los alumnos, el contexto sociocultural, las necesidades de aprendizaje y los objetivos de enseñanza ha encontrado el camino allanado en el contexto académico que nos ocupa. La tarea de la profesora es, por tanto, aprovechar esta sinergia, con miras a un trabajo y aprendizaje a largo plazo.

Se acuerda que los programas irán enfocados, principalmente, a la difusión de la lengua y culturas hispanas, teniendo en cuenta a una audiencia, en principio, india. Esto permitirá a los alumnos reflexionar sobre el aprendizaje de E/LE a partir del contraste con las lenguas y culturas de su país. De cara a los oyentes, se deberá recurrir a traducciones y aclaraciones que faciliten la comprensión de los contenidos.

Antes de empezar a diseñar el programa de radio, el primer paso es que los alumnos se familiaricen con la terminología del medio y con el formato del guion. Para ello, se presentan dos proyectos de programas radiofónicos (escaleta y audio), a modo de ejemplo. Seguidamente, se realiza una encuesta cualitativa individual sobre las expectativas de aprendizaje. Reflexionan por escrito un total de nueve alumnos. Citamos, a continuación, las respuestas relacionadas con los objetivos que se desean alcanzar con la tarea, indicando el número de referencias:

- 4 referencias: Mejorar la pronunciación; desarrollar destrezas comunicativas para participar en conversaciones en español, y adquirir fluidez.

- 3 referencias: Aprender vocabulario y trabajar en equipo.

- 2 referencias: Hablar más español en clase; aprender español más allá de los libros; aprender español de una forma más entretenida, y difundir el español en India y también aspectos culturales, como la comida y las fiestas.

- 1 referencia: repasar diálogos y vocabulario; contextualizar frases en diferentes situaciones; mejorar la gramática; hablar con entonación española; enseñar, en el futuro, inglés a hablantes hispanos o ser trabajadora social en Estados Unidos; enseñar español a los que no conocen el idioma y aprender para ser profesora de español en India; entender los diferentes usos de las palabras y evitar

\footnotetext{
${ }^{2}$ Ver http://chutneyconsalsa.blogspot.com.es/p/radio.html
} 
traducciones literales; conocer mejor la cultura española; introducir nuestra cultura a los españoles; conocer a personas de otros países a través de la radio; aprender cómo funciona una emisora de radio; aprender a escribir y a hablar para el medio radiofónico; mejorar destrezas orales para la radio; mejorar la modulación de la voz; aprender a hablar en público; superar el miedo escénico, y aprender de forma diferente.

La estructura general del programa ¿Salsa o salsa? acordada en clase es la siguiente:

Bloques: Un primer bloque ofrecerá contenidos relacionados con el aprendizaje de E/LE. De él se encargarán los alumnos de segundo curso. Seguidamente, un segundo bloque tratará temas culturales y de civilización, y lo realizarán las alumnas de tercer curso.

Lenguas: Español, inglés e hindi serán las lenguas de comunicación.

Audiencia: Estudiantes y amas de casa. En primer lugar, se considera la emisión desde Radio MUST. La audiencia a través del blog se cuenta como suma posterior.

Estilo: Animado, divertido y con música.

Franja horaria de emisión: Alrededor de las $14 \mathrm{~h}$ y de $19 \mathrm{~h}$ a $21 \mathrm{~h}$.

Duración: Alrededor de 20 minutos por programa, divididos en 10 minutos para el bloque de lengua y 10 minutos para el bloque de cultura.

El ciclo de creación de cada programa ha sido el siguiente:

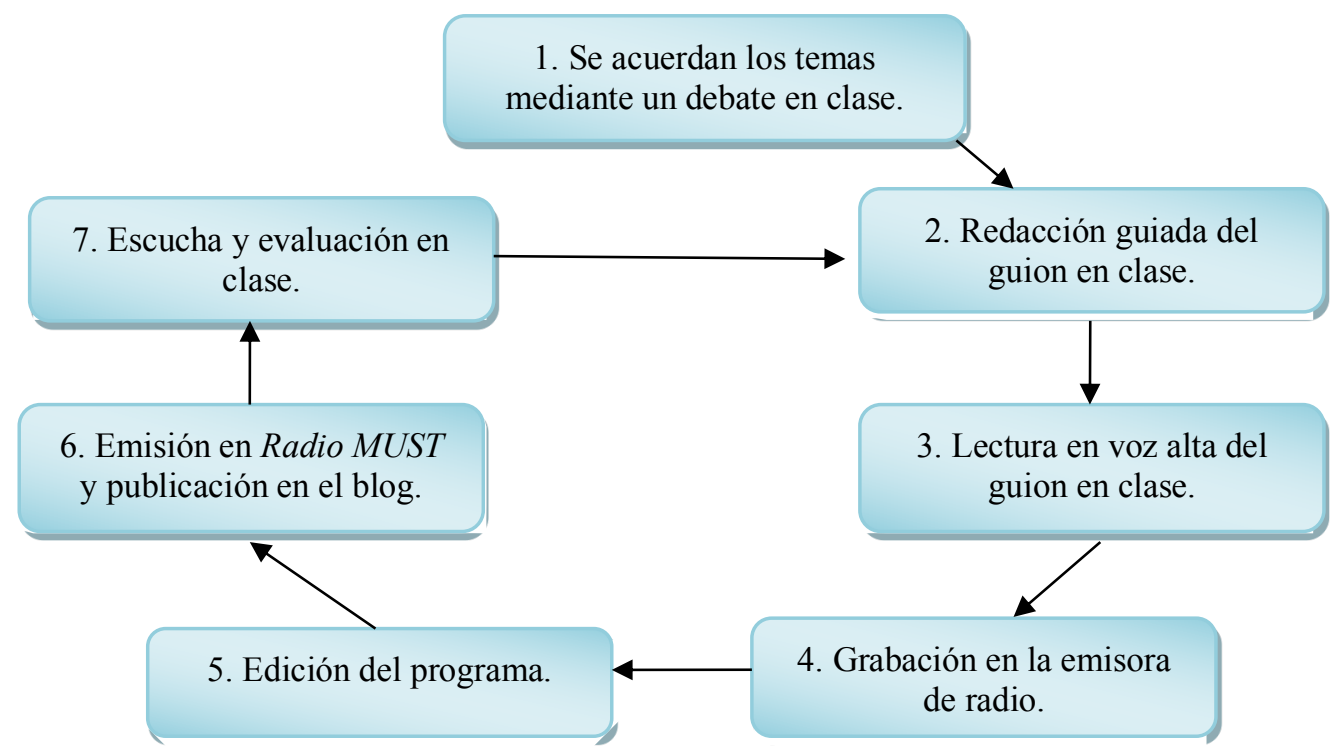

Gráfica 1. Ciclo de creación de los programas de radio

Tras cada programa, continuamos únicamente con la clase rutinaria de E/LE, antes de empezar el ciclo de nuevo, ya en el segundo paso, para la creación del programa siguiente. Se cree necesaria esta pausa porque las tareas radiofónicas suponen un elevado grado de concentración y un gran esfuerzo por parte de los alumnos. De esta manera, no les sobrecargamos, ya que esto podría tener un efecto contraproducente en el 
proceso de aprendizaje. Finalmente, al terminar el curso académico, los alumnos han reflexionado, por escrito, sobre el proceso individual de aprendizaje del idioma.

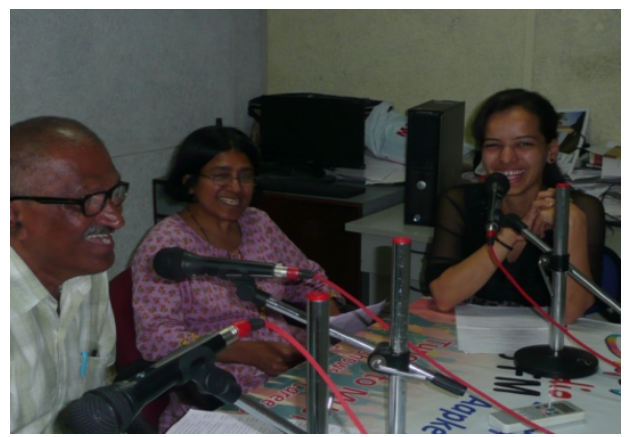

Imagen 1. Alumnos en la sala de grabación de Radio MUST

\section{Resultados y discusión}

Los temas de los cuatro programas de radio creados en el primer bloque de lengua giran en torno a los saludos, las despedidas, las formas de presentarse, de preguntar y dar información personal y, por último, las conversaciones en el contexto del restaurante. El vocabulario relacionado, una de las principales expectativas de aprendizaje para los alumnos, está contextualizado en las veinte nociones específicas clasificadas en el Plan Curricular del Instituto Cervantes (2007) para los niveles A1 y A2.

Los guiones se han elaborado a partir de materiales que los alumnos han trabajado previamente y que, por tanto, les resultan familiares. Esto ha aumentado la motivación $\mathrm{y}$, además, ha servido para repasar diálogos y vocabulario. Asimismo, el hecho de tratarse de situaciones comunicativas planificadas y preparadas antes de producirse ha facilitado la comunicación oral posterior.

Respecto al uso de la subordinación sintáctica en los discursos orales planificados (Briz 2016), destacamos su reducción al mínimo en los discursos radiofónicos, de menor complejidad sintáctica por naturaleza. Esto se acentúa en contextos de aprendizaje de idiomas con alumnos de niveles iniciales como el nuestro. Sí que se ha prestado mayor atención al uso de marcadores discursivos, al tratarse de un discurso más elaborado que los discursos orales espontáneos, como podemos constatar en los audios.

En el paso hacia la oralidad, partimos de textos escritos que los alumnos leen marcando las pausas de la voz. Se les anima a que trabajen la entonación y el acento enfático, y a que se ayuden de expresiones faciales y de movimientos de manos para reforzar el discurso y hacerlo más enérgico. Con ello, por un lado, mejoran la articulación y, por el otro, adoptan un estilo de locución propio. Siguiendo la clasificación de Briz (2016), el discurso está elaborado y ensayado, y tanto las relaciones personales como el contexto se negocian y determinan de antemano. Existe un mayor control de la producción y de la recepción y una minimización de las autorreparaciones durante la elaboración del discurso. No obstante, a pesar de recurrir a un léxico preparado previamente, la velocidad del discurso está condicionada por el nivel de lengua de los alumnos. Al escuchar los diálogos se percibe, con claridad, que no existe improvisación.

En la improvisación se debe destacar el buen manejo del idioma y la corrección sintáctica y gramatical, la propiedad en el uso de los términos y las expresiones idiomáticas, además de la 
claridad de expresión. El locutor debe tener una gran facilidad de palabra, cuidando la fluidez y continuidad de su parlamento. Debe poseer a su vez la capacidad de estructurar en forma lógica los pensamientos y expresarlos de manera atractiva a la radioescucha.

Blanch y Lázaro (2010: 191)

Entendemos, por tanto, que esta afirmación tan característica del lenguaje radiofónico no se hace extensiva a un contexto de trabajo como el nuestro, en el que el dominio del idioma es la meta y no el punto de partida. Resulta pertinente, por tanto, dejar este aspecto claro a los alumnos participantes desde el principio.

En cuanto a las pausas, con todo el significado que estas aportan, exceden los tres segundos máximos que recomiendan Blanch y Lázaro (2010) para el discurso radiofónico. Esto se sobreentiende, ya que se suele poseer menos fluidez en una lengua extranjera y, además, el nivel de español de los alumnos es básico. Se aprecia, sin embargo, el cambio de ritmo en el discurso cuando hablan en hindi y en inglés, lenguas que dominan y que alternan con el español para garantizar la comprensión del mensaje por parte de oyentes que puedan desconocer esta última.

Trabajar las pausas, como puntuación de las palabras habladas y que, por tanto, aporta significado al discurso, ha requerido una labor concienzuda, al no tratarse de hablantes nativos. Así, primero, nos hemos ayudado de ellas y de los signos de puntuación para delimitar unidades entonativas que los alumnos han marcado sobre el guion, con ayuda de la profesora, durante las actividades previas a la locución. A continuación, se ha practicado la lectura con el guion estructurado mediante pausas.

En general, al tratarse de un texto preparado de antemano, sin improvisación, las pausas se planifican en la misma línea que los contenidos lingüísticos. Como elemento a favor, hay que considerar la fase de postedición, la cual permite a los alumnos realizar pausas más largas durante la locución y prepararse para la frase siguiente, puesto que la duración de los silencios siempre podrá reducirse en la edición final posterior.

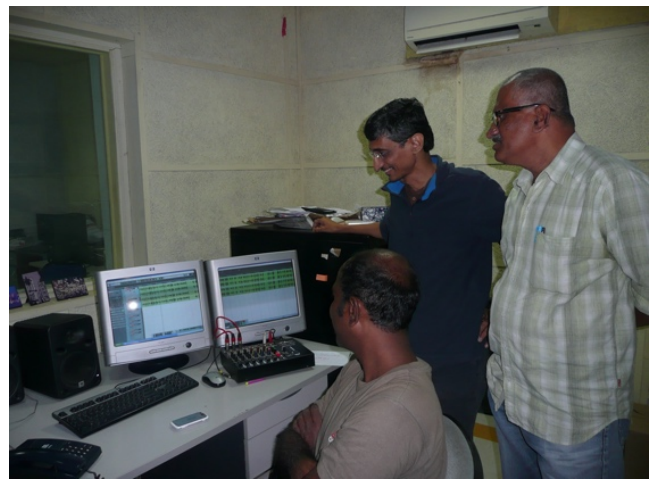

Imagen 2. Alumnos y técnico en la sala de control de grabación y edición de Radio MUST.

La modulación, la naturalidad en la expresión y la fluidez han sido retos que hemos tratado como hablantes no nativos. La labor previa de redacción y de ensayo de textos escritos formales y organizados les ha ayudado a comprenderlos bien y ha facilitado la expresión oral de un discurso que podríamos caracterizar como espontáneo ensayado. Debemos añadir que el hecho de ser el español un idioma en el que la correspondencia 
entre los fonemas y las grafías es bastante estrecha ha facilitado el aprendizaje tanto de la ortografía como de la pronunciación.

«I became more fluent in conversation and communication because of radio programs and I became more confident in speaking Spanish» (Seema Mansukhani, estudiante).

«When I started learning Spanish I was not much confident about speaking practice. Now I am confident in practicing language in oral and written» (Padmashree Sorate, estudiante).

En este ejercicio de redacción de guion y grabación del programa de radio, se han practicado destrezas de expresión e interacción escritas y orales de cara a una producción final. Se ha trabajado, además, la adecuación de la expresión y la interacción a las características del medio radiofónico, observando la pronunciación y la entonación en la expresión oral. Finalmente, se ha hablado con una audiencia en mente y esto ha supuesto un mayor esfuerzo por emitir un discurso claro y comprensible, pensando también que va a ser perdurable.

En general, y a pesar del nivel inicial de lengua de los alumnos, el conjunto del aprendizaje no ha resultado complicado, ya que se ha avanzado paso a paso y a partir de una planificación previa que ha tenido en cuenta situaciones de comunicación concretas. Se ha prestado especial atención a la hora de trasladar al estudio de grabación la entonación practicada durante las lecturas previas del guion en clase. Los alumnos carecen de hábitos de fijación en el uso de la lengua y, sin la ayuda de guía, tienden a la impulsividad a la hora de hablar. La práctica en el estudio, por tanto, ha sido una fase importante del aprendizaje, a pesar de haber ensayado antes el guion.

Llegados a este punto, cabe destacar el apunte mencionado por una alumna en la reflexión sobre el aprendizaje llevada a cabo al terminar el curso académico y antes de conocer los resultados de las pruebas formales:

«For me learning a language is more important than scoring marks in exams. Whatever my final marks will be, I am confident in practicing the language, both oral and written» (Padmashree Sorate, estudiante).

Deducimos, por tanto, la adecuación de considerar formas de evaluación en las que el aprendizaje no se mida solo mediante resultados finales, sino a partir de la observación de un proceso y evolución de los cuales los alumnos son conscientes.

Para concluir, en ausencia de un contexto social nativo de la lengua de estudio, observamos la utilidad de ofrecer a los alumnos herramientas que les permitan desarrollar la competencia comunicativa en el aula. Hay que seleccionar el input y la estrategia didáctica adecuados, explotarlos y adaptarlos a sus necesidades, así como observar y aprovechar los recursos disponibles para el aprendizaje. Este ha sido, en términos generales, el enfoque de nuestra práctica radiofónica.

\section{Conclusiones}

Este estudio de caso demuestra la conveniencia de insistir en el desarrollo de la competencia léxico-semántica paralelo al de la competencia pragmática, esta última de investigación menos voluminosa y más tardía que la primera pero igualmente importante en el trabajo de la competencia comunicativa. En el aula, de cara al 
desarrollo de competencias, los manuales de E/LE aportan información general que deberá adaptarse según el contexto. En este sentido, compartimos la idea de Rodríguez (2004) acerca de «convertirnos en investigadores de nuestra propia lengua»; en ser, más que consumidores de recursos, creadores de estos a la hora de trabajar la competencia pragmática en el aula, teniendo en cuenta el contraste cultural en cada momento.

Sobre el nivel de lengua adecuado, se suele hacer referencia a estudiantes de nivel avanzado o con una base más o menos sólida del idioma. Sustentamos aquí la opinión de Gómez (2004) sobre la adecuación de trabajar la competencia pragmática también en niveles inferiores. Con estos, como norma general, incidiríamos más en el lenguaje no verbal y en la utilización del espacio como complemento a un uso del lenguaje todavía limitado. Este estudio, sin embargo, demuestra que es igualmente viable prescindiendo de un lenguaje no verbal y de una información espacial visibles. El esfuerzo, cabe reconocer, es mayor con estudiantes de niveles inferiores.

Concluimos este artículo con dos extractos de las reflexiones posteriores de los alumnos que invitan a dejar una puerta abierta a futuras investigaciones sobre la relación que se establece entre la práctica de audición y la producción oral en el aprendizaje de lenguas extranjeras, sobre cómo se influyen y se determinan, así como el peso que el factor de la edad podría ejercer tanto en su desarrollo como en su combinación.

\footnotetext{
«The radio program was a good idea. I improved my pronunciation. More audio practice is still required in class, though» (Prakash Satam, estudiante mayor de 65 años).

«I could learn grammar well, composition and reading well, but I could not do so well in speaking. There were mainly two reasons for this: lack of practice during my first year of learning Spanish and my slight problem in hearing (maybe because of my age)» (Vivek Bhat, estudiante mayor de 65 años).
}

Con todo, la radio no solo permite llevar al aula muestras de lengua, de cultura y de sociedad, sino que, como hemos observado, también representa una ventana abierta al desarrollo de competencias comunicativas, de una forma más entretenida y más allá de los manuales, como demuestra el programa ¿Salsa o salsa? El resultado final ha sido una mejora visible de la competencia comunicativa general de los alumnos a lo largo del curso académico, una base sólida, también de autoconfianza, que les ha infundido mayor autonomía para tomar iniciativas de forma segura y más acertada a la hora de utilizar el idioma y de continuar sumando aprendizaje, dentro y fuera del aula.

\section{Bibliografía}

Blanch, Margarita y LÁZARO, Patrícia (2010). Aula de locución. Madrid: Cátedra.

BRIZ GÓMEZ, Antonio (2016). «Aportaciones del análisis del discurso oral». En J. Sánchez Lobato e I. Santos Gargallo (dirs.); Vademécum para la formación de profesores: Enseñar español como segunda lengua (L2)/lengua extranjera (LE) Tomo I (pp. 219-242). Madrid: SGEL.

ESCANDELl VIDAL, María Victoria (2004). Competencia comunicativa, arquitectura cognitiva y disciplinas lingüísticas. Universidad Nacional de Educación a Distancia.

ESCANDELl VIDAL, María Victoria (2016). «Aportaciones de la pragmática». En J. Sánchez Lobato e I. Santos Gargallo (dirs.); Vademécum para la formación de 
profesores: Enseñar español como segunda lengua (L2)/lengua extranjera (LE) Tomo I (pp. 179-197). Madrid: SGEL.

CASSANy I COMAS, Daniel (2016). «La expresión escrita». En J. Sánchez Lobato e I. Santos Gargallo (dirs.); Vademécum para la formación de profesores: Enseñar español como segunda lengua (L2)/lengua extranjera (LE) Tomo II (pp. 309-334). Madrid: SGEL.

GÓMEZ MORÓN, Reyes (2004). «La competencia pragmático-conversacional en los manuales de español como lengua extranjera: Breve revisión teórico-práctica desde la teoría pragmática de la cortesía lingüística». En Rushtaller, S. y Lorenzo, F. (ed.); La competencia lingüística y comunicativa en el aprendizaje del ELE (pp. 141-154). Sevilla: Edinumen.

Instituto Cervantes (2007). Plan curricular del Instituto Cervantes. Niveles de referencia para el español A1 y A2. Madrid: Biblioteca Nueva.

LANDONE, Elena (2009). «Reflexiones sobre la cortesía verbal en la enseñanza/aprendizaje de ELE», en MarcoELE, 8. Recuperado el 21/07/18, de https://marcoele.com/descargas/8/landone_cortesia.pdf

Ministerio de Educación, Cultura y Deporte (2002). Marco Común Europeo de Referencia para las lenguas: aprendizaje, enseñanza, evaluación. Madrid: Secretaría Técnica del MECD y Grupo Anaya.

RODRÍGUEZ, Silvia (2004). «Actividades para la enseñanza de pragmática en español como L2: el caso de los actos de habla». En Actas selectas del VI Congreso de didáctica del español. Indianapolis: Indiana University-Purdue University. 